\title{
Impedance of a Monopole Antenna With a Radial-Wire Ground System on an Imperfectly Conducting Half- Space, Part II
}

\author{
S. W. Maley and R. J. King \\ Contribution from Electrical Engineering Dept., University of Colorado, Boulder, Colo.
}

(Received August 23, 1963)

\begin{abstract}
The impedance of a vertical monopole located over the surface of an imperfectly conducting earth and having a radial-wire ground system has been studied theoretically by J. R. Wait. An experimental investigation discussed in part I of this paper gave results in good agreement with Wait's theory. This part of the paper is a presentation of calculations based on Wait's theory showing the behavior of antenna impedance as a function of antenna height, top-loading and number, size, and length of radial wires.
\end{abstract}

\section{Introduction}

A vertical monopole antenna over an imperfectly conducting ground excites currents in the ground near the base of the antenna which results in a substantial loss of radiated power. To reduce this power loss and thereby increase the efficiency of the radiating system, a highly conducting concentric circular disk may be placed at the ground surface. For economic reasons, the conducting disk is usually replaced by a system of radial wires. Such a system is not as effective as the disk but can be made nearly so by use of a sufficient number of wires.

The effectiveness of the radial wire system in reducing the ground losses ${ }^{1}$ was investigated theoretically and experimentally by observing its effects on the antenna base impedance. Part I of this paper [Maley and King, 1962] gave the results of this investigation as a function of the number of radial wires using the ground system diameter as a parameter. The investigation indicated reasonable agreement between the measured values of impedance and those calculated from formulas given by J. R. Wait [1954]. Measurements and calculations were made for frequencies of 4.20 and $9.60 \mathrm{G} / \mathrm{s}$ and for a ground plane of water. The success of Wait's formulas in predicting the effect of the radial wire ground system upon the monopole base impedance for these cases was considered sufficient justification to extend the calculations to other frequencies and ground parameters.

In this paper an analysis is made of the effects of monopole height, top-loading and number, length and size of the radial wires on the antenna impedance. Calculated values of impedance are plotted as functions of the various ground system and antenna

1 For the configuration considered, the ground losses were primarily $H$-field losses [Wait, 1958]. The calculations in this paper consider only these losses. parameters. These calculations extend those published by Wait and Pope [1955] who employed both graphical and analytical methods. The effect of ground parameters and frequency will be considered in part III of this paper.

\section{Theory}

The impedance of a monopole antenna over an imperfectly conducting ground has been given by Wait $[1954]$ as $^{2}$

$$
Z=Z^{\infty}-\frac{2 \pi}{I_{0}^{2}} \int_{0}^{\infty} E_{r}(r, 0) H_{\varphi}^{\infty}(r, 0) r d r
$$

$Z^{\infty}$ is the monopole impedance which would exist if the ground were perfectly conducting, $H_{\varphi}^{\infty}(r, 0)$ is the magnetic field at the surface of the ground for the same condition and with an antenna base current of $I_{0} . E_{r}(r, 0)$ is the radial component of the electric field at the surface of the imperfectly conducting ground with the radial wire system in place for an antenna base current of $I_{0}$. The time dependence is $\exp (i \omega t)$ where $\omega$ is angular frequency, $t$ is time and $i=\sqrt{-1}$. The coordinate system is shown in figure 1.

In most practical antenna systems the boundary conditions at the surface of the ground may be approximated to a good order of accuracy by the relation

$$
E_{r}(r, 0) \cong-\eta H_{\varphi}(r, 0) \cong-\eta H_{\varphi}^{\infty}(r, 0),
$$

where $H_{\varphi}(r, 0)$ is the magnetic field at the surface of the imperfectly conducting ground, and $\eta$ is the surface impedance which, in general, is a function

\footnotetext{
${ }_{3}$ The rationalized MKS system of units is used throughout.
} 


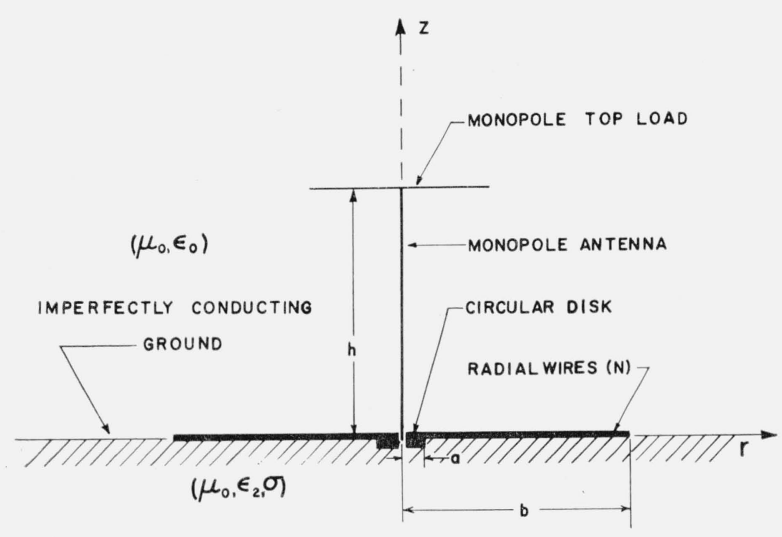

FiguRE 1. Sketch of antenna system.

of $r$. Equation (2) assumes $r H_{\varphi}^{\infty}(r, 0)$ varies slowly in a distance $\left|\frac{1}{\gamma}\right|$ where $\gamma=\left[i \mu \omega\left(\sigma+i \omega \epsilon_{2}\right)\right]^{1 / 2}$ is the propagation constant of the ground. Using (2), (1) may be written

$$
Z \cong Z^{\infty}+\frac{2 \pi}{I_{0}^{2}} \int_{0}^{\infty} \eta\left[H_{\varphi}^{\infty}(r, 0)\right]^{2} r d r .
$$

In the case of a circular conducting disk ground system, $\eta=0$ for $0<r<a$ where " $a$ " is the radius of the disk. Then (3) becomes

$$
Z \cong Z^{\infty}+\frac{2 \pi}{I_{0}^{2}} \int_{a}^{\infty} \eta_{g}\left[H_{\varphi}^{\infty}(r, 0)\right]^{2} r d r
$$

where $\eta_{g}$ is the effective surface impedance of the ground.

The wires of a radial wire ground system are normally connected to a small metal disk at the base of the antenna as shown in figure 2 . Then (3) becomes

$$
Z \simeq Z^{\infty}+\frac{2 \pi}{I_{0}^{2}} \int_{a}^{\infty} \eta_{e}(r)\left[H_{\varphi}^{\infty}(r, 0)\right]^{2} r d r
$$

where $\eta_{e}(r)$ is the surface impedance of the ground with the system of radial wires in place.

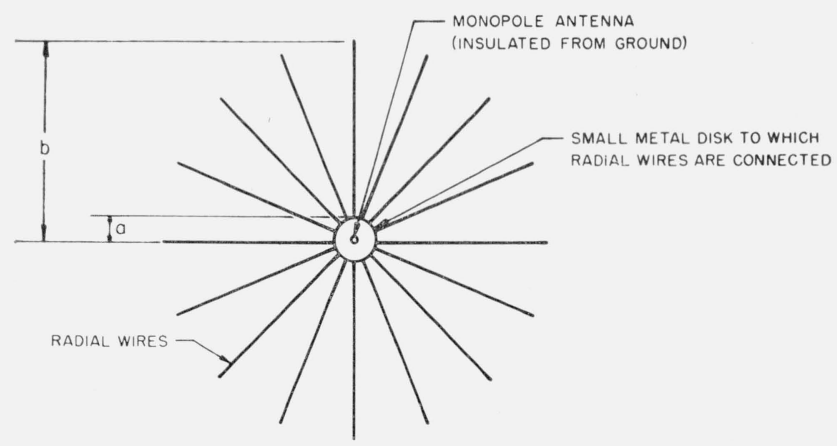

Figure 2. Top view of radial wire ground system.
It is interesting to compare the behavior of the impedance for the circular conducting disk ground system with that for the radial wire system. If $\eta_{g}$ in (4) is taken as the surface impedance of the imperfectly conducting ground, $\Delta Z=Z-Z^{\infty}$ may be calculated as a function of disk radius. Typical results as plotted on a rectangular impedance chart are shown in figure 3.

Now consider a radial wire ground system in which the radials are connected to a small disk of radius " $a$ " and extend to radius " $b$ ". The base impedance as a function of the number of radial wires and given value of " $b$ " for such a system is shown typically as the dashed curve $A B$ on figure 3 . When no radial wires are present the impedance is the same as that for a conducting disk of radius " $a$ ". As the number of radials increases without limit for a constant value of " $b$ ", the impedance should approach that for a disk of radius " $b$ ". 'Thus, the curve of impedance as a function of the number of radials $N$ should start at point $A$ and end at point $B$ in figure 3. Quantitative data on this variation and a comparison between calculated and measured values has been given in part I of this paper.

The approximate variation of the impedance between these two points may be found from (5) and from the relation [Wait, 1954; Maley and King, $1962]$

where

$$
\eta_{e}=\frac{\eta_{g} \eta_{w}}{\eta_{g}+\eta_{w}}, \quad a<r<b,
$$

$$
\begin{gathered}
\eta_{w}=\frac{i 2 \pi \eta_{0} r}{\lambda N} \ln \left(\frac{r}{N c}\right), \\
\eta_{g}=\left[\frac{i \mu \omega}{\sigma+i \omega \epsilon_{2}}\right]^{1 / 2}
\end{gathered}
$$

$\eta_{0}$ is the intrinsic impedance of free space and $c$ is the radius of the wire.

The parallel impedance assumption (6) ignores mutual effects between ground and grid and assumes radial symmetry as well as perfect electrical contact between the wires and the earth. Experimental evidence [Smith and Devaney, 1958; 1959] indicates that mutual coupling and a potential difference can exist between the wires and earth, but may be neglected in (6) for a large number of wires and high soil conductivity. Gustafson, Devaney, and Smith [1959] give an approximate evaluation of these losses, as well as copper losses.

Equation (7) assumes $\left|\gamma_{\epsilon} d\right|<<1$ where $d=2 \pi r / N$ is the spacing between wires and $\gamma_{e}$ is the propagation constant for a thin wire in the interface and is given by [Wait, 1954]

$$
\gamma_{e}=\left[\frac{\gamma_{0}^{2}+\gamma^{2}}{2}\right]^{1 / 2} .
$$

If calculations are made for the impedance as a function of the number of radials $N$ for several different values of wire lengths, the results may be 


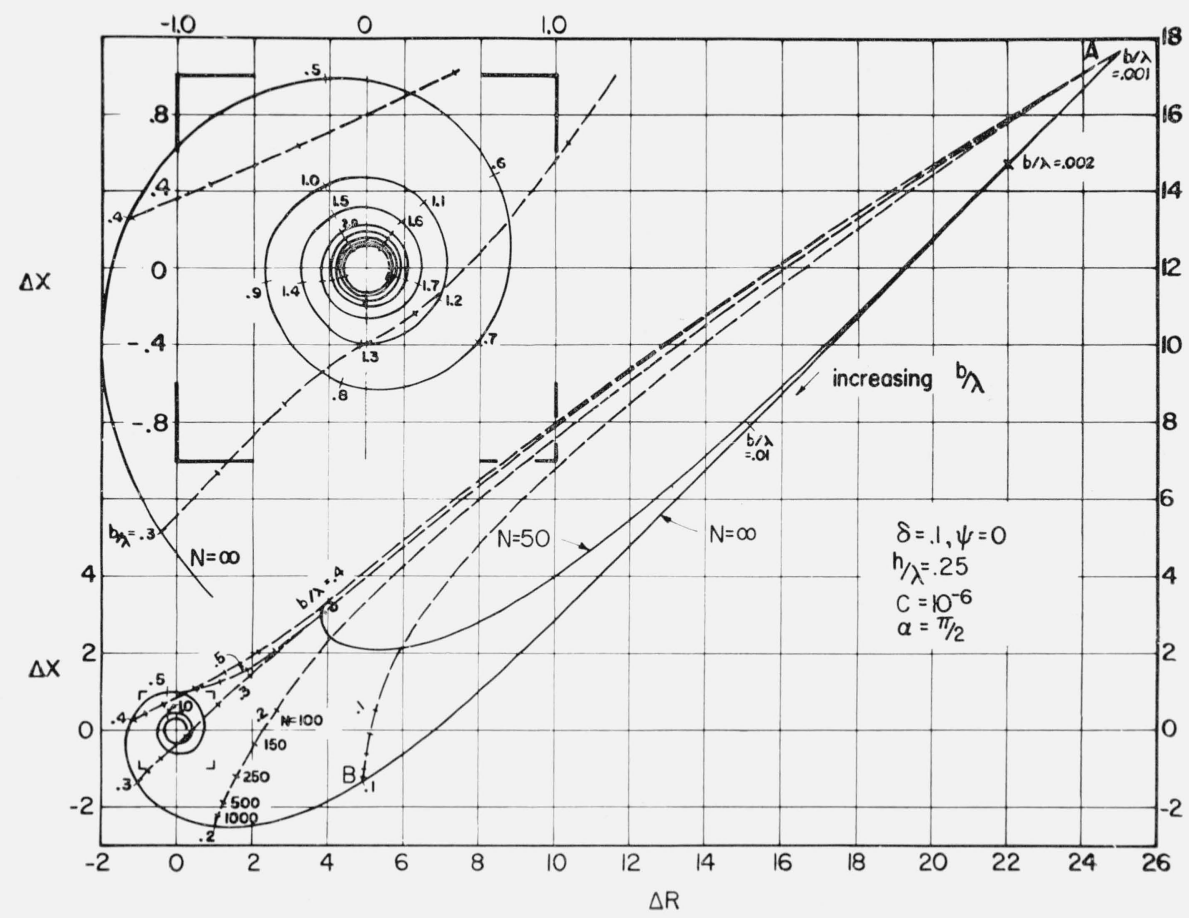

$\Delta x$

Figure 3. Monopole base impedance loci showing the effect of varying $\mathrm{N}$ and system radius.

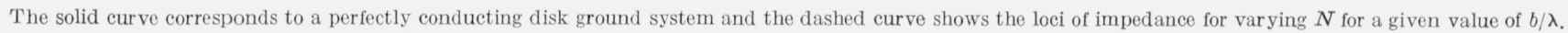
The inset shows an expansion of the region of convergence of the solid spiral.

shown as the family of dashed curves on figure 3 . Each starts at point $A$ corresponding to a small disk of radius " $a$ ", and terminates at a point corresponding to the given value of " $b$ ". From this figure the variation of impedance $\Delta Z$ as a function of " $b$ " is apparent. A curve connecting the points representing the same number of radials but of varying length has the shape of a spiral similar to that for the conducting disk ground system of varying diameters. As $N$ decreases, the spirals become smaller and closer to point $A$, and as $N$ increases, the spirals become nearly coincident with the spiral corresponding to a solid disk.

The change of impedance with respect to an infinite perfectly conducting plane (the limit of the spiral as $b \rightarrow \infty$ ) may be obtained from (5), (6), (7), and (8). The result is

$$
\begin{aligned}
\Delta Z \cong \frac{2 \pi}{I_{0}^{2}} \int_{a}^{b} \eta_{e}(r)\left[H_{\varphi}^{\infty}(r, 0)\right]^{2} r d r & \\
& +\frac{2 \pi}{I_{0}^{2}} \int_{b}^{\infty} \eta_{g}\left[H_{\varphi}^{\infty}(r, 0)\right]^{2} r d r .
\end{aligned}
$$

\section{Calculations}

The magnetic field, $H_{\varphi}^{\infty}(r, 0)$ for a perfectly conducting ground can be found if the current distribution on the monopole is known. It can usually be assumed that this distribution is sinusoidal and is given by

$$
I(z)=I_{0} \frac{\sin (\alpha-\beta z)}{\sin \alpha}
$$

where $\beta=2 \pi / \lambda, \quad \alpha=\beta\left(h+h^{\prime}\right), \quad \lambda$ is the free space wavelength, $h$ is the actual monopole height, and $h^{\prime}$ is a parameter specifying the top-loading of the monopole. For this choice $H_{\varphi}^{\infty}(r, 0)$ is given by Wait (1954) as

$$
\begin{aligned}
H_{\varphi}^{\infty}(r, 0)= & \frac{i I_{0}}{2 \pi \sin \alpha}\left[\frac{e^{-i \beta \rho}}{r} \cos (\beta h-\alpha)\right. \\
& \left.\quad-\frac{e^{-i \beta r}}{r} \cos \alpha+\frac{i h e^{-i \beta \rho}}{r \rho} \sin (\beta h-\alpha)\right]
\end{aligned}
$$

where $\rho=\left(r^{2}+h^{2}\right)^{1 / 2}$. Equation (12) applies sufficiently well near a monopole antenna with symmetrical top loading.

Calculations were made from (10) for a variety of parameters. In most applications, the normalized radius of the wire $C=c / \lambda$ varies between $0.05 \times 10^{-6}$ $\leq C \leq 20 \times 10^{-6}$. The theoretical calculations do not account for copper loss. These may be estimated by other means. Themonopole height varies between $0.003 \leq h / \lambda \leq .25$ and the number of radials lies in the range $50 \leq N<\infty$. The top-loading parameter, $\alpha$, lies between $2 \pi h / \lambda \leq \alpha \leq \pi / 2$, the lower and upper limits corresponding to $\overline{h^{\prime}}=0$ and full top-loading, respectively. The permittivity $\epsilon_{2}$ and conduc- 
tivity $\sigma$ of the imperfectly conducting earth are accounted for in the parameter $\delta$ which may be defined in terms of $\eta_{g}$;

$$
\eta_{g}=\left[\frac{i \mu \omega}{\sigma+i \omega \epsilon_{2}}\right]^{1 / 2}=\sqrt{i} \eta_{0}\left[\frac{\left(\delta^{\prime}\right)^{2}}{1+i\left(\delta^{\prime}\right)^{2} \epsilon}\right]^{1 / 2}=\sqrt{i} \eta_{0} \delta
$$

where $\epsilon=\epsilon_{2} / \epsilon_{0}$ is the relative dielectric constant, and

Then

$$
\delta^{\prime}=\left(\frac{\omega \epsilon_{0}}{\sigma}\right)^{1 / 2} .
$$

and

$$
\delta=\delta^{\prime}\left[\frac{1}{1+i\left(\delta^{\prime}\right)^{2} \epsilon}\right]=|\delta| e^{-i \psi}
$$

$$
\psi=\frac{1}{2} \tan ^{-1}\left[\left(\delta^{\prime}\right)^{2} \epsilon\right] .
$$

If $\left(\delta^{\prime}\right)^{2} \epsilon<<1$ then $\psi \cong 0$ which corresponds to highly conducting earth and negligible displacement currents. The ranges of $\delta$ and $\psi$ were chosen to be $0.01 \leq \delta \leq 0.30$ and $0 \leq \psi \leq \pi / 4$. Part II of this paper is mainly concerned with the variation of $N, C, h / \lambda$, and $\alpha ; \delta$ and $\psi$ will be considered in part III.

\section{Conclusions}

Figures 4 through 10 reveal nearly the same conclusions as those made by Wait. Essentially the same formulation was used here except that Wait assumed $\alpha=\pi / 2$. This corresponds to $h / \lambda=0.25$ [Wait, 1954] or a uniform current distribution for short monopoles where $h / \lambda \leq 0.1$ [Wait, 1955]. Where the results can be compared good agreement exists with the curves in the present papers.

Figure 4 shows the effect of varying the number of radials $N$ and antenna height $h / \lambda$, for $\delta=0.10$ and $\psi=0$, and wire radius to wavelength ratio $C=c / \lambda=$ $10^{-6}$. These curves show that, in general, there is little to be gained by making $N$ greater than approximately $250 . \Delta Z$ is evidently a rapidly varying function for small $b / \lambda$, whatever the values of the parameters although $\Delta X$ generally varies less rapidly with $b / \lambda$ than $\Delta R$ does. Figure $4 \mathrm{c}$ shows that $\Delta X$ varies over a much larger range with $N$ than $\Delta R$ does for small $h / \lambda$. This is to be expected since the imaginary part of $Z^{\infty}$ is very large for short monopoles. The rate of change of both $\Delta R$ and $\Delta X$ for small $b / \lambda$ is extremely rapid for short antennas and small $b / \lambda$, although the curves are not considered accurate when $b / \lambda$ is less than the earth skin depth due to reflections from the ends of the radials. Also, the boundary condition (2) assumes $r H_{\varphi}^{\infty}(r, 0)$ varies slowly in a distance $\left|\frac{1}{\gamma}\right|$.

Larsen [1962] has investigated the ratio between the $E$-field and $H$-field losses per unit area for a monopole of various heights, current distributions, and top-loading using a radial-wire ground system. It was shown that for short antennas the $E$-field losses are comparable or larger than the $H$-field losses especially for large $N$, and increase somewhat with top-loading. Heavy ground cover such as snow, vegetation, etc., increases the $E$-field losses even more. Gustafson, Devaney, and Smith [1959] give a method of approximating these cover losses. For higher antennas with sinusoidal current distribution, the $E$-field losses become negligibly small.
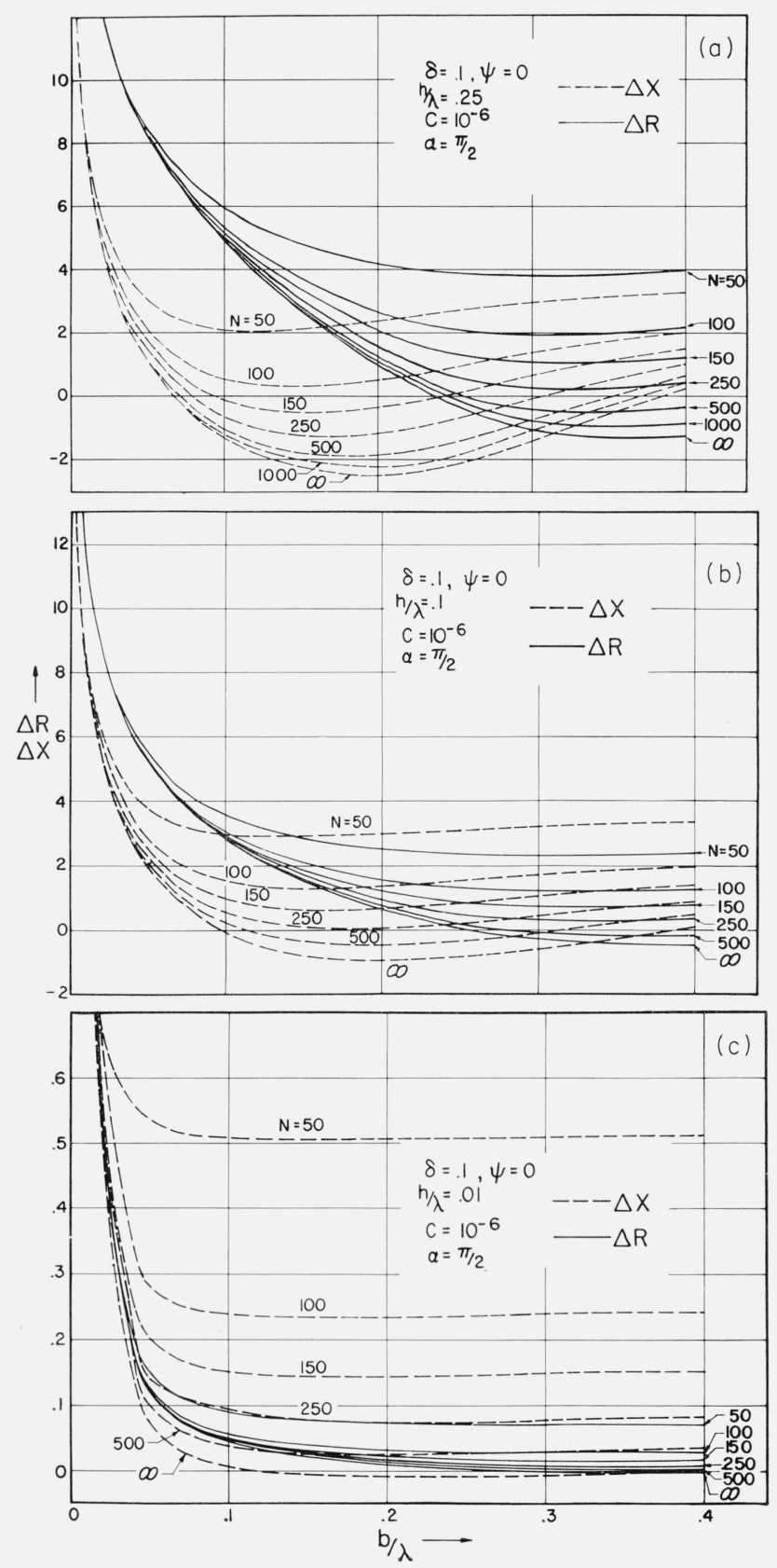

Figure 4. a, b, and c. $\Delta \mathrm{R}$ and $\Delta \mathrm{X}$ versus length of radial wires for select d values of $\mathrm{N}$ and $h / \lambda$ and parameters as specified. 


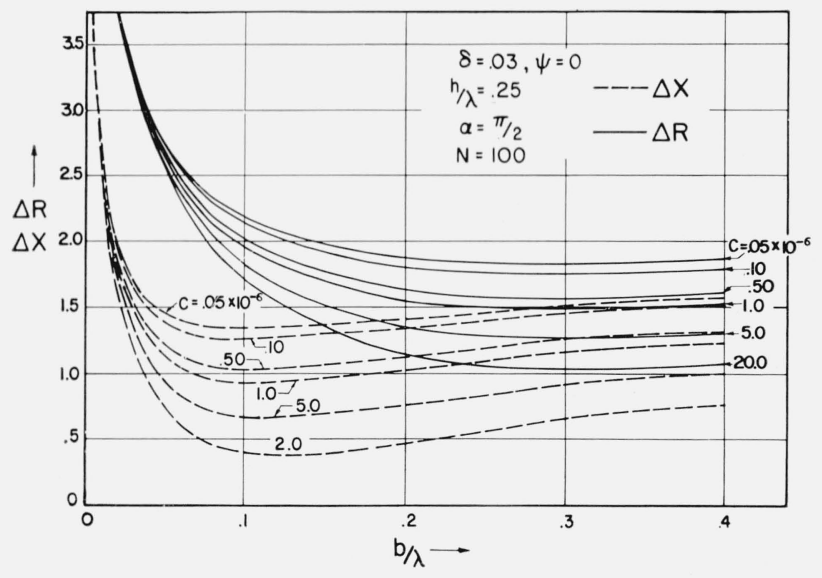

FIgURE 5. $\Delta \mathrm{R}$ and $\Delta \mathrm{X}$ versus length of radial wires for parameters as specified for selected values of $\mathrm{C}$.

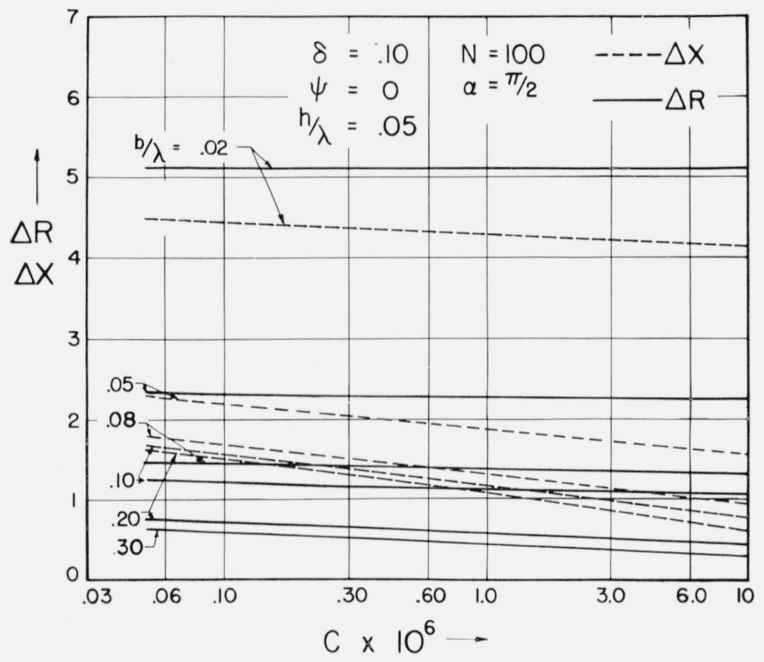

FIgURE 6. $\Delta \mathrm{R}$ and $\Delta \mathrm{X}$ versus normalized wire radius $\mathrm{C}$ for selected values of $\mathrm{b} / \lambda$ and parameters as specified.

Equation (7) is not expected to be valid when $\left|\gamma_{e} d\right|$ is comparable to or greater than unity. If $|\gamma|>>\left|\gamma_{0}\right|$, then

$$
\left|\gamma_{e} d\right|=\frac{(2 \pi)^{2}}{N} \frac{r}{\lambda} \sqrt{\frac{1}{2} \frac{\epsilon_{2}}{\epsilon_{0}} \mid 1-\frac{i \sigma}{\omega \epsilon_{2}}} \mid
$$

Thus, for example, if $\epsilon_{2} / \epsilon_{3}\left|1-\frac{i \sigma}{\omega \epsilon_{2}}\right|=10$ and $N=50$, the results are not expected to be correct for $b / \lambda$ greater than about 0.20 . However, part I of this paper shows that this restriction may be relaxed somewhat. Comparison of figures $4 \mathrm{a}$, b, and c clearly shows that the necessary length of radials decreases significantly for short antennas.

Figure 5 shows that changing the wire size by a factor of 200 for the indicated parameters changes $\Delta R$ and $\Delta X$ by less than $1 \mathrm{ohm}$ and for very small values of $b / \lambda$ the wire size has practically no effect. This is even more apparent in figure 6 where it is seen that the ground system radius affects $\Delta R$ and
$\Delta X$ much more significantly than $C$ does, and that $\Delta X$ is affected much more than $\Delta R$. For these reasons, in nearly all of the calculations, the value of $C$ was taken to be $10^{-6}$ which corresponds to $\mathrm{B}$ and S No. $8(0.327 \mathrm{~cm}$ diameter $)$ at $183 \mathrm{kc} / \mathrm{s}$ and B and $\mathrm{S}$ No. $4(0.518 \mathrm{~cm}$ diameter $)$ at $115 \mathrm{kc} / \mathrm{s}$. The value of $C$ should be primarily determined so that the wires will be large enough to prevent breakage during installation, but not so large as to be wasteful of copper.

'The effects of varying the top-loading parameter $\alpha$ and antenna height $h / \lambda$ are shown in figures 7 and 8 . As expected, these curves exhibit an increasing $\Delta R$

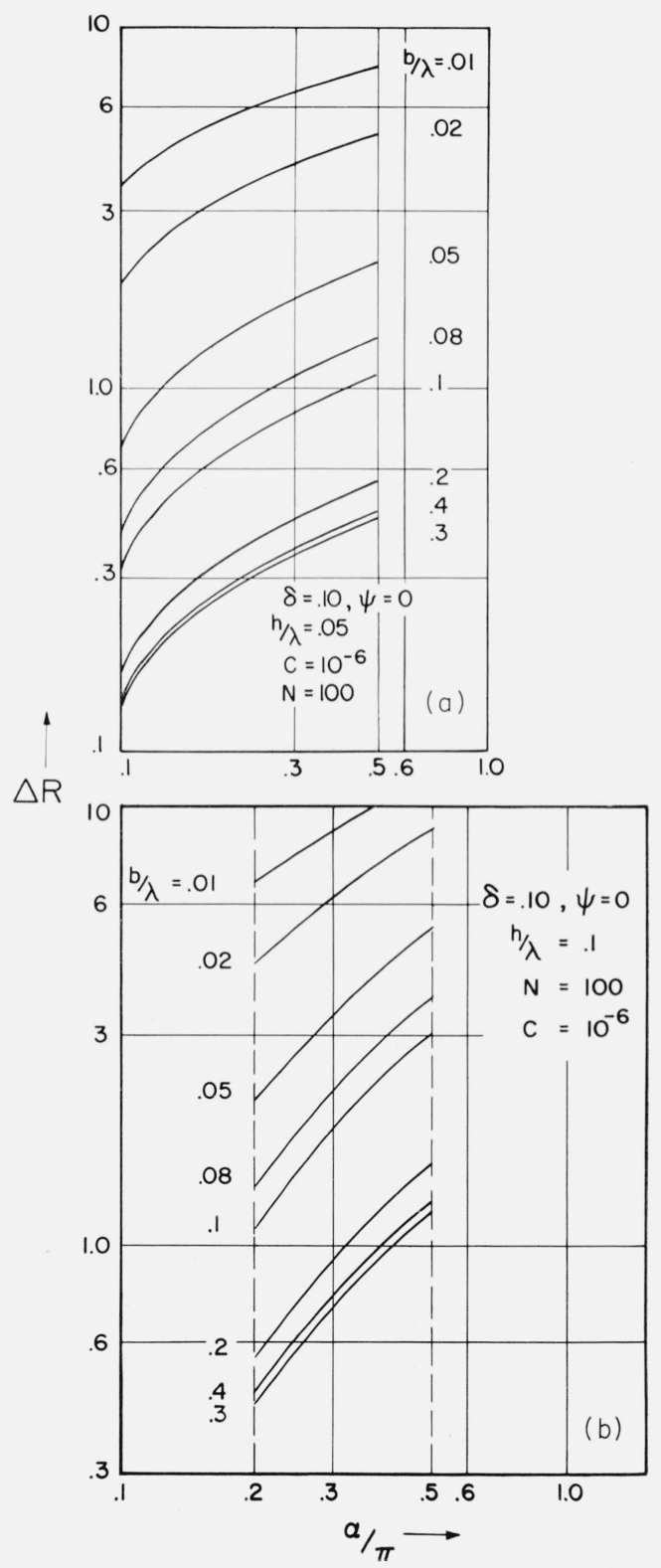

Figure 7. a and b. $\Delta \mathrm{R}$ versus top-loading parameter $\alpha$ for selected values of $\mathrm{b} / \lambda$ and parameters as specified.

(a) $h / \lambda=0.05$, (b) $h / \lambda=0.10$. 

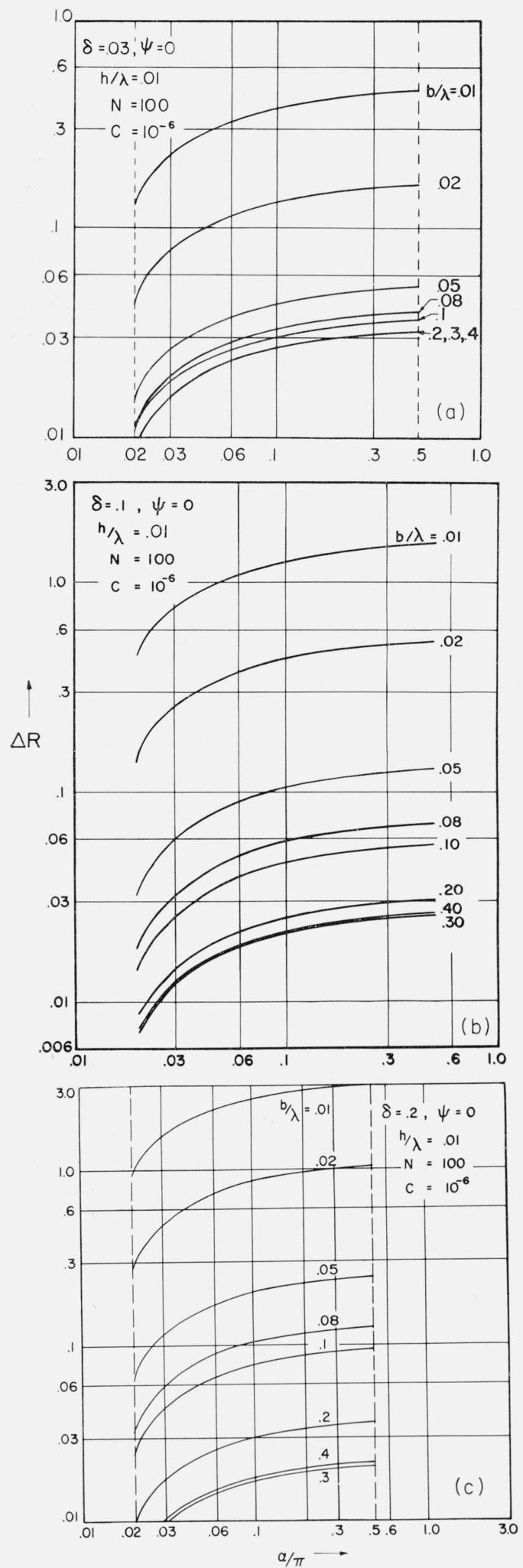

Figure 8. a, b, and c. $\Delta \mathrm{R}$ versus top-loading parameter $\alpha$ for selected values of $\mathrm{b} / \lambda$ and parameters as specified.

(a) $\delta=0.03$, (b) $\delta=0.1$, (c) $\delta=0.2$. for increased top-loading, due to the increased radiated power. It is also obvious that increasing the ground system radius causes a considerable decrease of $\Delta R$ due to decreased ground losses; however, increasing $b / \lambda$ to values greater than 0.2 has very little effect upon $\Delta R$. Comparison of figures $7 \mathrm{a}, \mathrm{b}$, and $8 \mathrm{~b}$ shows how the impedance increases significantly for all values of top-loading with increasing antenna height. A similar effect is achieved as $\delta$ is increased as may be seen from figures $8 \mathrm{a}, \mathrm{b}$, and $\mathrm{c}$; however, this increase in $\Delta R$ as $\delta$ increases for all values of top-loading is due to increased ground losses. Decreasing $h / \lambda$ and $|\delta|$ corresponds to smaller spirals on the rectangular chart of figure 3 .

Negative $\Delta R$ does not necessarily signify that the system is more efficient than a perfectly conducting ground system. However, in general, the longer the radials the more efficient the system. Normally, ground systems are designed to operate in a region where $\Delta R$ decreases with increasing " $b$ " and in this region it is generally said that the antenna system efficiency increases with decreasing $R$.

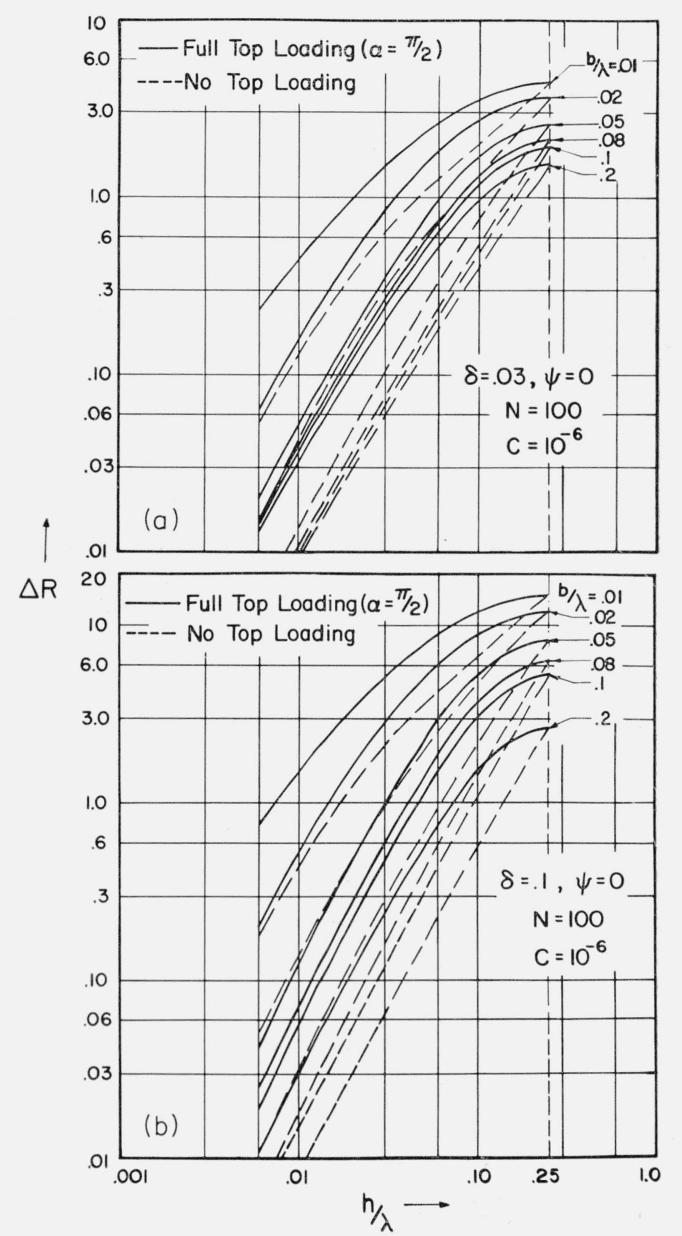

Figure 9. a and b. $\Delta \mathrm{R}$ versus antenna height $\mathrm{h} / \lambda$ for the two cases of full top-loading and unloaded monopoles for parameters as specified.

(a) $\delta=0.03$, (b) $\delta=0.10$. 


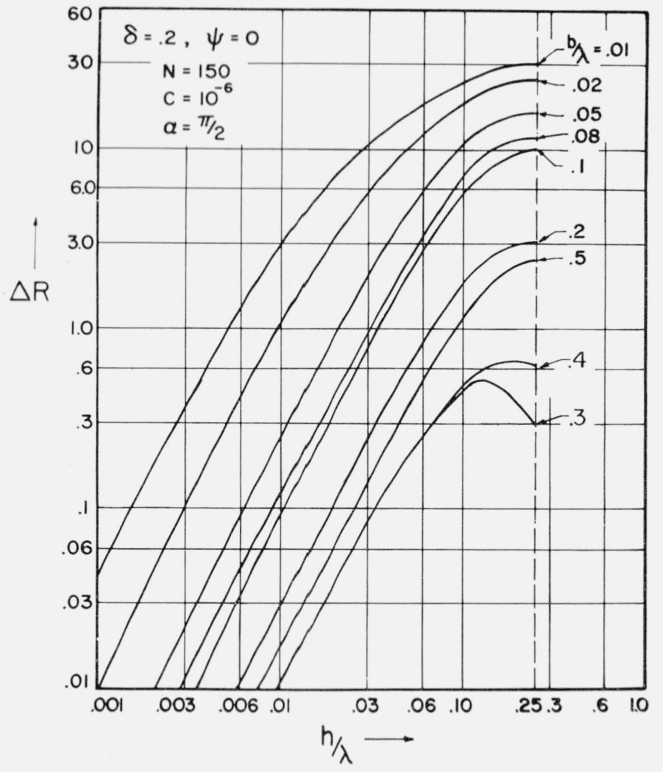

Figure 10. $\Delta \mathrm{R}$ versus antenna height $\mathrm{h} / \lambda$ for full top-loading and parameters as specified.

Figure 9 illustrates the effect of varying the antenna height for a given set of system parameters for fully loaded and unloaded monopoles. The advantage of using top-loading for $h / \lambda$ less than 0.25 is quite significant as may be seen from figures 10a and b. The near linear log-log relationship makes it possible to write an empirical formula for $\Delta R$ where the curves are sufficiently linear, e.g., for $b / \lambda=0.1$ and the parameters of figure $9 \mathrm{~b}$,

$$
\Delta R=K\left(\frac{h}{\lambda}\right)^{k}=72.4\left(\frac{h}{\lambda}\right)^{1.818}
$$

is valid for $h / \lambda$ less than 0.2 .

Wait and Pope [1955] proposed that if the radiation resistances are equal for unloaded and fully loaded monopoles, then they are electrically equivalent in length. It was shown that for the fully loaded monopole of heights $0.025,0.050$, and 0.10 wavelength, the same $\Delta R$ is obtained for unloaded monopoles of heights $0.050,0.095$, and 0.175 wavelength, respectively. Figures $9 \mathrm{a}$ and $\mathrm{b}$ are in good agreement with this conclusion. Figure 9 shows that for loaded monopoles of height $0.025,0.050$, and 0.10 wavelength have the same $\Delta R$ as unloaded monopoles of height
$0.053,0.104$, and 0.18 wavelength, respectively, except for small $b / \lambda$ where the values of the latter are slightly higher. In figure 10, one could sketch the behavior of the unloaded case fairly accurately by observing the behavior of figure 9 .

The authors express their appreciation to James R. Wait of the National Bureau of Standards Central Radio Propagation Laboratory for his constructive suggestions and to the laboratory itself for the use of their digital computer. This research was originally started under the support of the Electronics Directorate of the United States Air Force Cambridge Research Center (Contract No. AF 19(604)-4556), was continued through support of the University of Colorado Council on Research and was completed under National Bureau of Standards Contract No. CST-7429. Mr. King was performing independent study under a National Science Foundation Faculty Fellowship. Appreciation is also extended to Lillie C. Walters of the National Bureau of Standards Laboratories who initially programmed this problem, to L. R. Branch who completed the collection of computer data, and to the many others who correlated the data and drew the curves.

\section{References}

Gustafson, W. E., T. E. Devaney, and A. N. Smith (June 27, 1959), Study of North Atlantic VLF Transmitting Station, NEL Report 909, Appendix A and C.

Larsen, Tove (Mar.-Apr. 1962), The E-field and H-field losses around antennas with a radial ground wire system, J. Res. NBS 66D (Radio Prop.) No. 2, 189-204.

Maley, S. W., and R. J. King (Mar-Apr. 1962), Impedance of a monopole antenna with a radial wire ground system on an imperfectly conducting half-space. Part I, J. Res. NBS 66D (Radio Prop.), No. 2, 175-180.

Smith, A. N., and T. E. Devaney (Feb. 1958), A study of currents in an electrically short ground system of few radials, NEL Techn. Memo. 272

Smith, A. N., and T. E. Devaney (Sept.-Oct. 1959), Fields in elestrically short ground system, an experimental study, J. Res. NBS 63D (Radio Prop.), No. 2, 175-180.

Wait, J. R., and W A. Pope (1954), The characteristies of a vertical antenna with a radial conductor ground system, Appl. Sci. Res B4 177-195. Note that in equation (5), $\sin (\beta h-\alpha)$ should be $\sin (\alpha-\beta h)$.

Wait, J. R., and W. A. Pope (May 1955), Input resistance of LF unipole aerials, Wireless Engr. 32, 131-138.

Wait, J. R. (1958), Calculations of transverse current loss in buried wire ground systems. Appl. Sci. Res. $7 \mathbb{B}$ 8186.

(Paper 68D2-328) 